\title{
ELN risk stratification and outcomes in secondary and therapy- related AML patients consolidated with allogeneic stem cell transplantation
}

\author{
Madlen Jentzsch $\mathbb{1}^{1} \cdot$ Juliane Grimm ${ }^{1} \cdot$ Marius Bill $\mathbb{1}^{1} \cdot$ Dominic Brauer $^{1} \cdot$ Donata Backhaus $^{1} \cdot$ Karoline Goldmann $^{1}$. \\ Julia Schulz ${ }^{1} \cdot$ Dietger Niederwieser $^{1} \cdot$ Uwe Platzbecker $^{1} \cdot$ Sebastian Schwind $^{1}{ }^{1}$
}

Received: 24 August 2020 / Revised: 8 October 2020 / Accepted: 2 November 2020 / Published online: 19 November 2020

(c) The Author(s) 2020. This article is published with open access

\begin{abstract}
Secondary or therapy-related acute myeloid leukemia (s/tAML) differs biologically from de novo disease. In general s/tAML patients have inferior outcomes after chemotherapy, compared to de novo cases and often receive allogeneic stem cell transplantation (HSCT) for consolidation. The European LeukemiaNet (ELN) risk stratification system is commonly applied in AML but the clinical significance is unknown in s/tAML. We analyzed $644 \mathrm{~s} / \mathrm{tAML}$ or de novo AML patients receiving HSCT. s/tAML associated with older age and adverse risk, including higher ELN risk. Overall, s/tAML patients had similar cumulative incidence of relapse (CIR), but higher non-relapse mortality (NRM) and shorter overall survival (OS). In multivariate analyses, after adjustment for ELN risk and pre-HSCT measurable residual disease status, disease origin did not impact outcomes. Within the ELN favorable risk group, CIR was higher in s/tAML compared to de novo AML patients likely due to a different distribution of genetic aberrations, which did not translate into shorter OS. Within the ELN intermediate and adverse group outcomes were similar in de novo and s/tAML patients. Thus, not all s/tAML have a dismal prognosis and outcomes of s/tAML after allogeneic HSCT in remission are comparable to de novo patients when considering ELN risk.
\end{abstract}

\section{Introduction}

Since acute myeloid leukemia (AML) is a biologically and clinically highly heterogeneous disease, a reliable risk stratification is very important to personalize treatment strategies. At diagnosis, the European LeukemiaNet (ELN) risk classification is a recommended risk stratification system, widely used, and has been shown to provide prognostic information in AML patients undergoing chemotherapy as well as allogeneic hematopoietic stem cell transplantation (HSCT) [1-3]. In addition, the evaluation of measurable

Supplementary information The online version of this article (https:// doi.org/10.1038/s41409-020-01129-1) contains supplementary material, which is available to authorized users.

Sebastian Schwind

Sebastian.Schwind@medizin.uni-leipzig.de

1 Medical Clinic and Policlinic 1, Hematology, Cellular Therapy and Hemostaseology, Leipzig University Hospital,

Leipzig, Germany residual disease (MRD) allows the adjustment of risk stratification during disease course [1,4]. Over the last years, a growing incidence of patients with secondary (sAML) or treatment-related (tAML) AML has been observed [5, 6]. This comes as a result of the demographic changes with higher life expectancies as well as better cancer treatment options with an increasing number of patients surviving their primary neoplasm [6, 7]. The growing need for understanding s/tAML to improve risk stratification and subsequently patients' outcomes is hampered by the low proportion of patients treated within clinical studies as compared to de novo cases [5, 8]. Regarding the associated prognosis, data on the rate of patients achieving a complete remission (CR) remain inconclusive with similar CR rates for de novo and tAML patients in a German analysis [7], but lower CR rates for s/tAML patients in Danish and Swedish registry data [8,9] and another German study [2]. After consolidation chemotherapy, shorter disease free and overall survival (OS) have been observed for s/tAML compared to de novo cases [7-11]. The adverse outcomes of s/tAML were also suggested to be independent from the higher incidence of adverse risk cytogenetics, especially in 
younger AML patients [7-9, 12]. As a consequence of the low cure rates of not more than $20 \%$ after chemotherapy alone [8, 9], allogeneic HSCT often is the preferred consolidation option in s/tAML patients. Here, mostly registrybased data not including de novo AML individuals suggest allogeneic HSCT as a suitable and often curative treatment option for s/tAML patients [13-18]. However, data comparing outcomes of s/tAML and de novo AML patients undergoing allogeneic HSCT remain sparse. A recent registry-based analysis by the EBMT on reduced intensity (RIC) or myeloablative conditioning (MAC) HSCT showed higher relapse rates, higher non-relapse mortality (NRM), and shorter OS in s/tAML compared to de novo AML patients [19]. In contrast, a monocentric study in which the majority of patients were younger and received MAC suggested comparable outcomes for s/tAML patients [20]. However, s/tAML patients are often older than individuals with de novo AML [7-9], and may not be candidates for MAC- or even RIC-HSCT, also due to comorbidities and previous treatments. In addition, both studies did not report outcomes in the context of the current ELN risk classification, or the MRD status prior to HSCT, which both have been shown to impact patients' outcomes [3, 21]. Here, we report outcomes of mostly older patients receiving allogeneic HSCT at our institution within the context of the most recent ELN risk classification.

\section{Subjects and methods}

\section{Patients and treatment}

We retrospectively analyzed 644 consecutive AML patients, who received an allogeneic HSCT at the University of Leipzig at a median age of 59.7 years (range 16.3-76.8 years). For all patients, associations of the disease origin with baseline clinical and genetic factors were assessed ("association set"). Of those, 534 patients were transplanted in $\mathrm{CR}$ or $\mathrm{CR}$ with incomplete peripheral recovery (CRi) and included in the outcome analysis ("outcome set"). Conditioning regimens in the 534 patients in the outcome set were either MAC $(n=142,27 \%)$, RIC $(n=13,2 \%)$ or NMA $(n=379, n=71 \%)$. RIC conditioning was applied within the MC-FludT.14/L trial (EudraCT Number 2008-002356-18). Reasons for NMAHSCT as opposed to MAC-HSCT were age over 50 years if receiving unrelated $\mathrm{HSCT}$ and over 55 years if receiving related HSCT, prior autologous HSCT $(n=7)$ or active infections $(n=8)$. All patients received G-CSF-stimulated peripheral blood stem cells as graft source. Stem cell donors were human leukocyte antigen (HLA) matched related $(n=$ $121,23 \%)$, HLA matched unrelated $(n=306,57 \%)$ or had
Table 1 Clinical and genetic characteristics for all patients according to disease origin (de novo vs secondary or treatment related), $n=644$.

\begin{tabular}{|c|c|c|c|c|}
\hline & $\begin{array}{l}\text { All patients } \\
n=644\end{array}$ & $\begin{array}{l}\text { De novo } \\
\text { AML } \\
n=416\end{array}$ & $\begin{array}{l}\text { Secondary or } \\
\text { treatment-related AML } \\
n=228\end{array}$ & $P$ \\
\hline Age at diagnosis, years & & & & $<0.001$ \\
\hline Median & 59.0 & 56.0 & 62.1 & \\
\hline Range & $14.3-76.5$ & $14.3-76.5$ & 27.1-74.7 & \\
\hline Sex, $n(\%)$ & & & & 0.05 \\
\hline Male & 334 & $204(49)$ & $130(57)$ & \\
\hline Female & 310 & $212(51)$ & $98(43)$ & \\
\hline Hemoglobin, g/dL & & & & 0.69 \\
\hline Median & 8.9 & 9 & 8.9 & \\
\hline Range & $3.2-15.7$ & $3.2-15.7$ & $5.4-15$ & \\
\hline Platelet count, $\times 10^{9} / \mathrm{L}$ & & & & 0.25 \\
\hline Median & 63 & 65 & 59 & \\
\hline Range & $1-950$ & $2-950$ & $1-547$ & \\
\hline WBC, $\times 10^{9} / \mathrm{L}$ & & & & 0.01 \\
\hline Median & 6.5 & 8.6 & 5.3 & \\
\hline Range & $0.1-432$ & $0.5-385$ & $0.1-432$ & \\
\hline Blood blasts, $\%$ & & & & $<0.001$ \\
\hline Median & 20 & 24 & 12 & \\
\hline Range & $0-98$ & $0-98$ & $0-97$ & \\
\hline BM blasts, $\%$ & & & & $<0.001$ \\
\hline Median & 50 & 60 & 35 & \\
\hline Range & $0-100$ & $0-100$ & $0-95$ & \\
\hline BM CD34+/CD38- burden, \% & & & & $<0.001$ \\
\hline Median & 0.7 & 0.5 & 1.1 & \\
\hline Range & $0-89$ & $0-75$ & $0-89$ & \\
\hline Normal karyotype, $n(\%)$ & & 0.006 & & \\
\hline Absent & 329 & $192(52)$ & $137(63)$ & \\
\hline Present & 259 & $180(48)$ & $79(37)$ & \\
\hline $\begin{array}{l}\text { ELN2017 genetic risk group, } \\
n(\%)\end{array}$ & & & & $<0.001$ \\
\hline Favorable & 114 & $97(33)$ & $17(12)$ & \\
\hline Intermediate & 129 & $84(28)$ & $45(32)$ & \\
\hline Adverse & 195 & $115(39)$ & $80(56)$ & \\
\hline$N P M 1, n(\%)$ & & & & $<0.001$ \\
\hline Wild type & 345 & $217(71)$ & $128(86)$ & \\
\hline Mutated & 111 & $90(29)$ & $21(14)$ & \\
\hline CEBPA, $n(\%)$ & & & 0.73 & \\
\hline Wild type & 326 & $232(88)$ & $94(87)$ & \\
\hline Mutated & 45 & $31(12)$ & $14(14)$ & \\
\hline FLT3-ITD, $n(\%)$ & & & & $<0.001$ \\
\hline Absent & 358 & $224(72)$ & $134(90)$ & \\
\hline Present & 103 & $88(28)$ & $15(10)$ & \\
\hline FLT3-TKD, $n(\%)$ & & & & 0.003 \\
\hline Wild type & 379 & $248(87)$ & $131(96)$ & \\
\hline Mutated & 42 & $37(13)$ & $5(4)$ & \\
\hline$R U N X 1, n(\%)$ & & & & 1 \\
\hline Wild type & 95 & $61(85)$ & $34(85)$ & \\
\hline Mutated & 17 & $11(15)$ & $6(15)$ & \\
\hline$A S X L 1, n(\%)$ & & & & 0.41 \\
\hline Wild type & 95 & $63(88)$ & $32(80)$ & \\
\hline Mutated & 17 & $9(13)$ & $8(20)$ & \\
\hline TP53, $n(\%)$ & & & & 1 \\
\hline Wild type & 99 & $64(89)$ & $35(88)$ & \\
\hline Mutated & 13 & $8(11)$ & $5(13)$ & \\
\hline
\end{tabular}

ASXL1 additional sex combs-like 1 gene, $B M$ bone marrow, $B A A L C$ brain and acute leukemia, cytogenetic gene, CEBPA CCAAT/enhancer-binding protein alpha gene, ELN European LeukemiaNet, FLT3-ITD internal tandem duplication of the FLT3 gene, $H b$ hemoglobin, $M N 1$ meningioma 1 gene, NPM1 nucleophosmin 1 gene, $P B$ peripheral blood, RUNX1 Runt-related transcription factor 1 gene, TP53 tumor protein 53 gene, WBC white blood count. 
Fig. 1 AML patients in the association set $(n=644)$. a Distribution of disease origin and $\mathbf{b}$ distribution of the ELN risk groups according to disease origin.

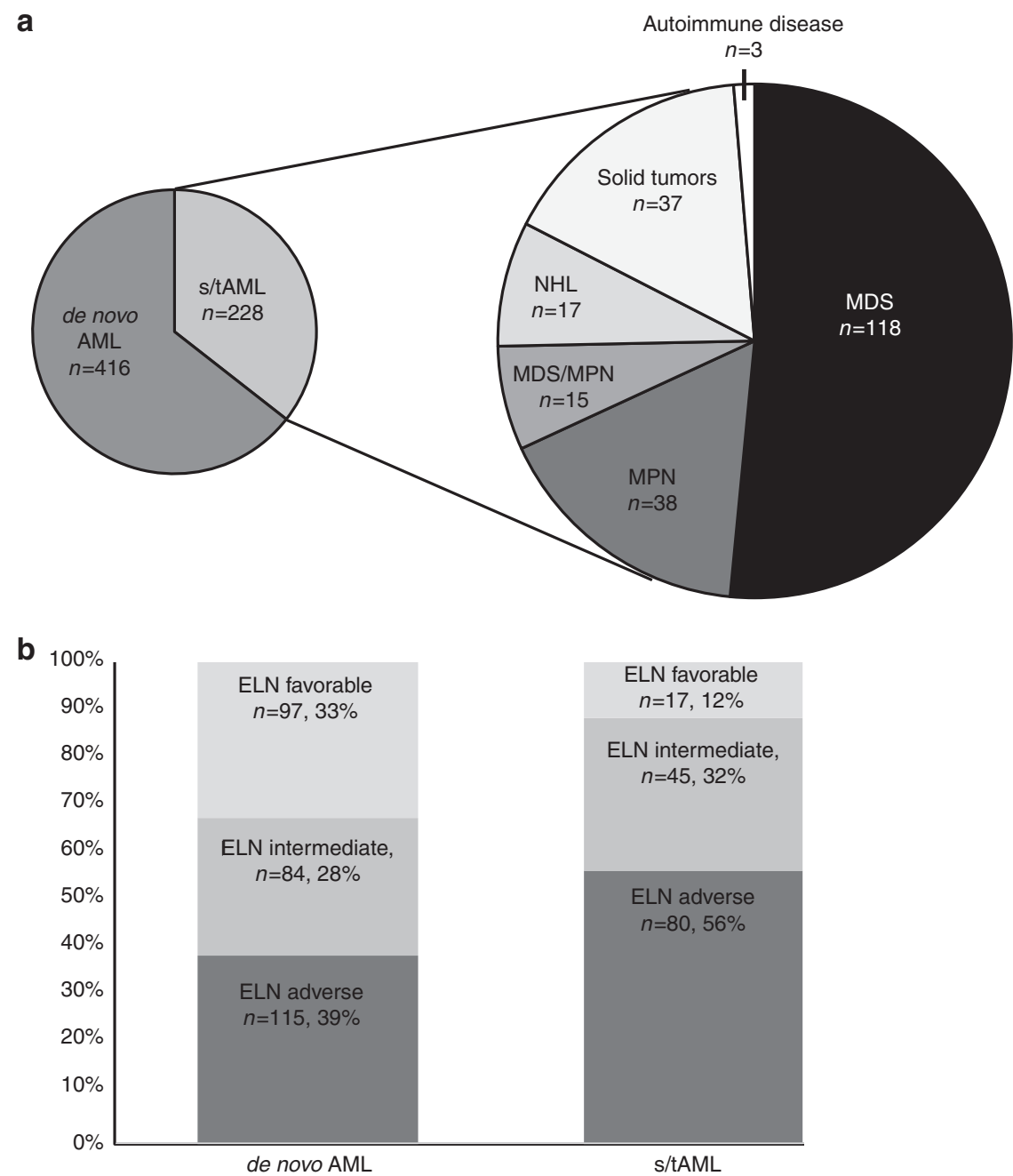

at least one HLA mismatch $(n=107,20 \%)$. Prior to allogeneic HSCT, patients received age-dependent standard cytarabine-based chemotherapy protocols. As the reported patients received chemotherapy prior to the approval of a liposomal combination of cytarabine and daunorubicin (CPX-351) in Europe, none of the here analyzed s/tAML patients received the substance. Details on the applied therapies are given in the Supplementary Information. Further patients' characteristics are shown in Table 1 and Supplementary Tables S1-S3. Median follow-up after HSCT was 3.7 years for patients alive. Written informed consent was obtained from all patients in accordance with the Declaration of Helsinki.

\section{Definitions of secondary or treatment-related AML}

sAML was defined as AML developing after an antecedent myeloid neoplasm, i.e., myelodysplastic syndrome (MDS), myeloproliferative neoplasm (MPN), or MDS/ MPN. tAML was defined as AML developing after exposure to chemotherapy or radiation applied for the treatment of lymphomas, solid tumors, or autoimmune diseases [22].

\section{Cytogenetics, molecular marker, flow cytometry, and MRD}

Cytogenetic aberrations, surface antigen expression of common surface markers, mutations in the genes NPM1 and $C E B P A$ and the presence of FLT3-ITD were assessed in pretreatment bone marrow samples as previously described $[23,24]$. For patients with material available, the mutation status of 54 genes included in the TruSight Myeloid Sequencing Panel (Illumina) was evaluated at diagnosis as previously described [24, 25]. Patients were grouped according to the ELN2017 risk classification [1] according to the diagnostic cytogenetic and molecular data available. Determination of the leukemic stem cell population at diagnosis and pre-HSCT MRD status was performed as previously described [24, 26-28]. 


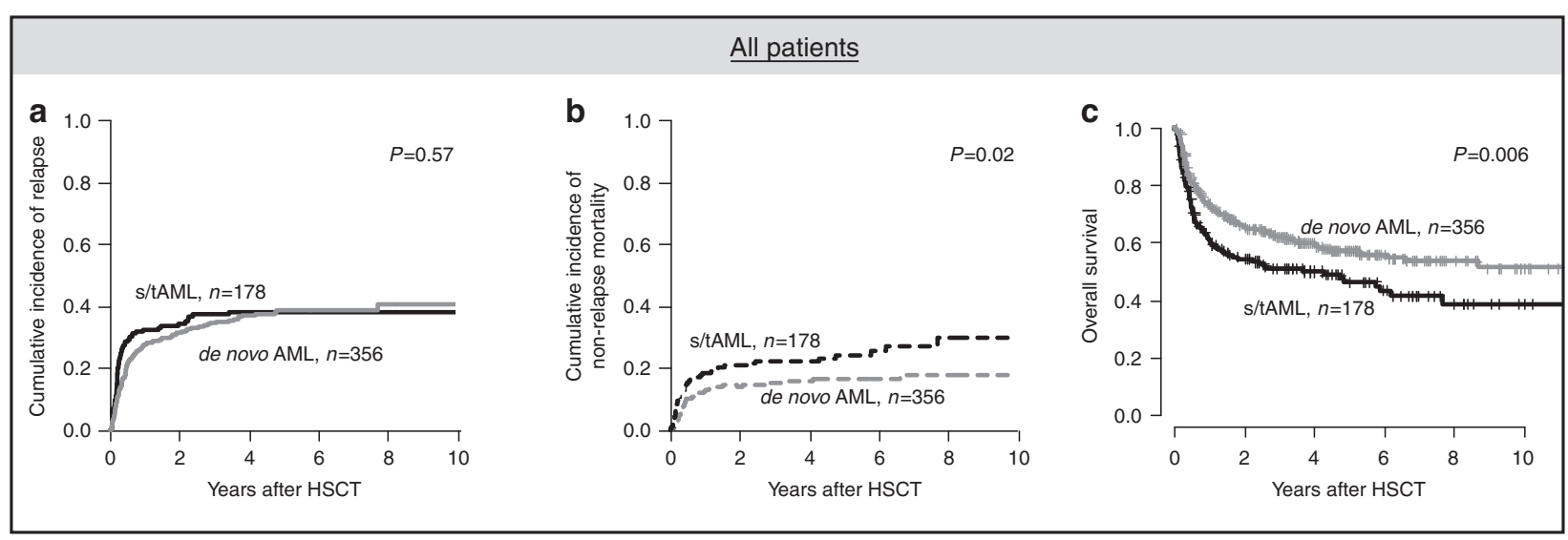

Fig. 2 Outcome according to disease origin (de novo vs secondary or treatment-related AML) for patients in the outcome set $(n=534)$. a Cumulative incidence of relapse, $\mathbf{b}$ non-relapse mortality, and $\mathbf{c}$ overall survival in all patients.

\section{Statistical analyses}

All statistical analyses were performed using the $\mathrm{R}$ statistical software platform (version 3.4.3) [29]. For further details see the Supplementary Information.

\section{Results}

\section{Incidence of $\mathbf{s} / \mathbf{t A M L}$}

Overall, 416 patients (64\%) had de novo AML while 171 patients $(27 \%)$ had sAML (18\% after prior MDS [MDSsAML], $2 \%$ after prior MDS/MPN, and $6 \%$ after prior MPN [MPN-sAML]) and 57 patients (9\%) had tAML (3\% after lymphoma, $6 \%$ after a solid tumor and $0.5 \%$ of patients after autoimmune disorders, Fig. 1a). Detailed information on the type of prior malignancies is given in the Supplementary Information. Median time from cytotoxic treatment to AML diagnosis in tAML patients was 4.5 years (range $0.5-22.3$ years).

\section{Characteristics of s/tAML patients}

Compared to de novo AML patients, s/tAML patients were older $(P<0.001$ and $P=0.006$, for sAML or tAML, respectively) and had a lower white blood count $(P=0.03$ and $P=0.05$, respectively) at diagnosis (Table 1 and Supplementary Table S1). In addition, there were lower bone marrow $(P<0.001)$ and peripheral blood blast percentages $(P<0.001)$ at diagnosis and more male patients in the sAML patient cohort $(P=0.003)$. s/tAML patients also had a higher $\mathrm{CD} 34+/ \mathrm{CD} 38-$ cell burden $(P<0.001$ and $P=$ 0.05 , respectively) and presented with a distinct immunophenotype (see Supplementary Information and Supplementary Table S2). s/tAML patients were more likely to have a del5/5q $(P=0.01$ and $P=0.01$, respectively $)$ and a del7/7q $(P=0.05$ and $P<0.001$, respectively) but less likely to have a core binding factor $(\mathrm{CBF})$ AML $(P<0.001$ and $P=0.01$, respectively), a normal karyotype $(P=0.05$ and $P=0.02$, respectively), or a FLT3-ITD $(P<0.001$ and $P=0.05$, respectively). In addition, patients with tAML more often had a complex $(P=0.006)[1]$ and a monosomal karyotype $(P<0.001)[30]$ while patients with sAML had a trend for more trisomy $8(P=0.06)$, were more likely to be SRSF2 mutated $(P=0.03)$ or JAK2 mutated $(P<0.001)$ but less likely to be NPMI $(P<0.001)$ or FLT3-TKD mutated $(P=0.001)$. Taken together, s/tAML patients were also by trend less likely to harbor a RAS pathway mutation (considering NRAS, KRAS, HRAS, and PTPN11 mutations, $P=$ $0.10)$. Regarding patients in the outcome set, sAML patients more often received a NMA conditioning $(P<0.001)$, were more likely to receive their allogeneic HSCT in first CR/ CRi $(P=0.03)$, more likely to have a CRi compared to a CR $(P=0.005)$, while we observed no difference in preHSCT MRD status between de novo and s/tAML patients $(P=0.78$, Supplementary Table S3). S/tAML patients were less likely to have a related donor $(P=0.009)$, by trend more likely to develop aGvHD $(P=0.06)$, while cGvHD was similar between s/tAML and de novo AML patients $(P=0.31)$. While tAML patients had a higher comorbidity index $($ HCT-CI) than de novo individuals $(P<0.001)$, the HCT-CI did not differ between de novo and sAML patients $(P=1)$. Importantly, s/tAML patients had a different distribution of the ELN risk groups compared to de novo disease and were more likely to harbor adverse ELN risk $(P<0.001$ and $P=0.01$, respectively, Fig. $1 \mathrm{~b})$,

\section{Outcome of s/tAML patients}

In the whole outcome set (Fig. 2), s/tAML patients had comparable CIR $(P=0.57)$ as de novo AML patients, but significantly higher NRM $(P=0.02)$ and shorter OS $(P=$ 0.006). However, patients receiving NMA-HSCT had 


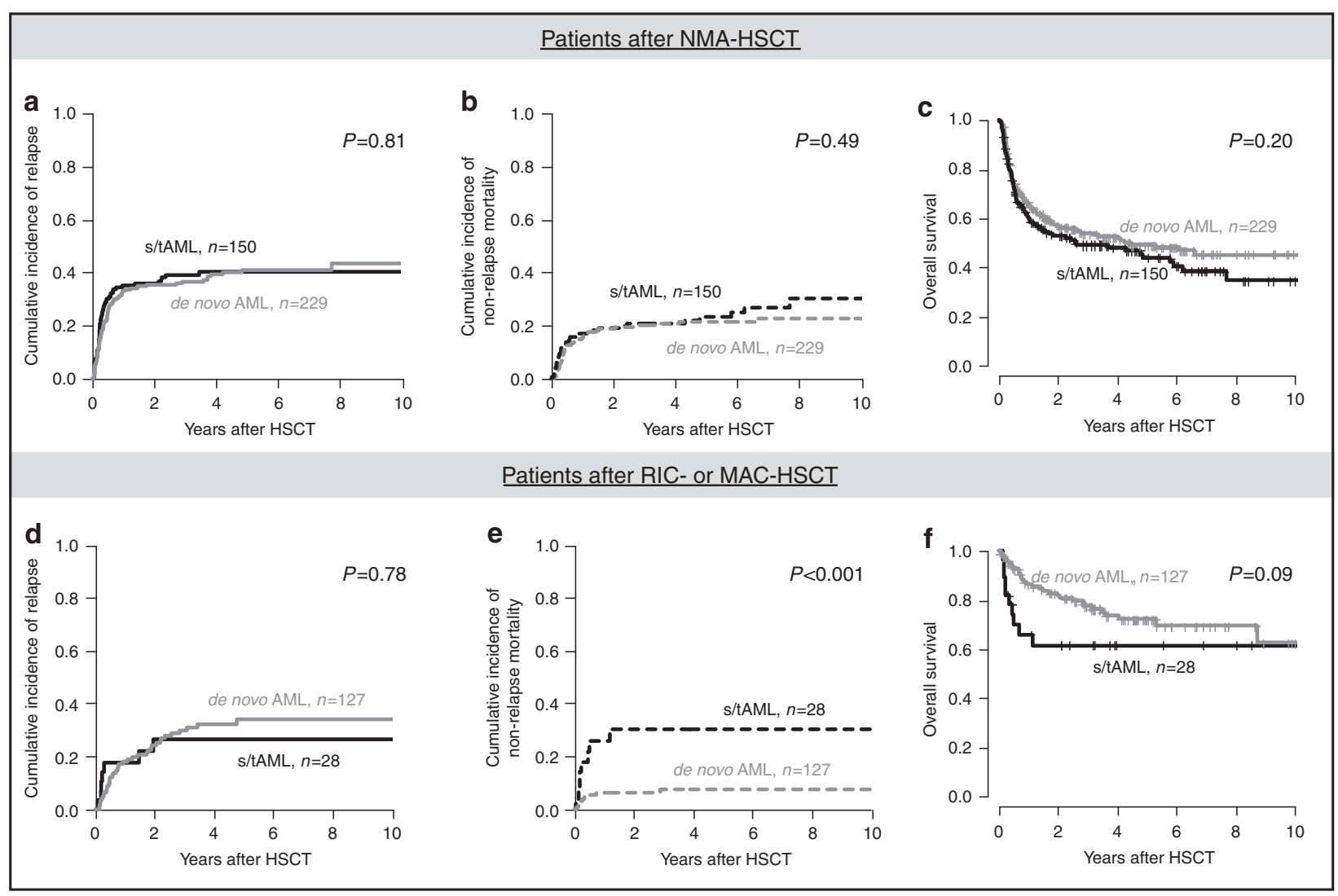

Fig. 3 Outcome according to disease origin (de novo vs secondary or treatment-related AML) for patients in the outcome set $(n=$ 534) given separately for both conditioning regimens. a Cumulative incidence of relapse, $\mathbf{b}$ non-relapse mortality, and $\mathbf{c}$ overall survival for

higher CIR $(P=0.02)$, higher NRM $(P=0.009)$, and shorter OS $(P<0.001)$ than patients receiving RIC- or MAC-HSCT (Supplementary Figs. S1 and S2) and a higher proportion of patients receiving NMA-HSCT had s/tAML $(P=0.03$, Supplementary Table S3), resulting in a potential bias for outcome analyses. Thus, outcome was analyzed separately for NMA conditioned and RIC or MAC conditioned patients. Restricting the analysis to patients receiving NMA-HSCT (Fig. 3a-c), there was no different CIR $(P=$ $0.81)$, NRM $(P=0.49)$, or OS $(P=0.20)$ between de novo and s/tAML patients. In contrast, in patients receiving RICor MAC-HSCT (Fig. 3e, f) with the caveat of limited patient numbers $(n=28)$ and a potential selection bias, s/tAML patients had a significantly higher NRM $(P<0.001)$, by trend shorter OS $(P=0.09)$ but similar CIR $(P=0.78)$. Similar results were observed when we restricted our analyses to patients transplanted in first CR/CRi (Supplementary Fig. S3).

In multivariate analyses for the whole patient cohort, s/ tAML patients did not have distinct outcome compared to patients with de novo AML while ELN risk and pre-HSCT MRD status remained significant factors for CIR and OS (Table 2). Multivariate analyses for patients receiving patients receiving NMA-HSCT $(n=379)$ and $\mathbf{d}$ cumulative incidence of relapse, e non-relapse mortality, and $\mathbf{f}$ overall survival for patients receiving RIC- or MAC-HSCT $(n=155)$.

NMA-HSCT or RIC- or MAC-HSCT separately is shown in Supplementary Table S4.

\section{Clinical and genetic characteristics of s/AML patients within the three ELN risk groups}

Distribution of de novo or s/tAML as well as of primary neoplasm within s/tAML patients differed between the three ELN risk groups and is depicted in Fig. 4a, e, i.

Within patients with favorable risk according to ELN, s/ tAML patients were older $(P=0.02)$ and had by trend a lower bone marrow blast percentage at diagnosis $(P=0.08)$ than de novo individuals. They were more likely to have a normal karyotype $(P=0.01)$ and to be NPM1 mutated $(P=$ $0.04)$, but less likely to harbor CBF AML $(P=0.003)$, or a FLT3-ITD $(P=0.04$, Fig. 4d, Supplementary Table S5). Within patients with intermediate risk according to ELN, s/ tAML patients were older $(P=0.02)$ and had lower bone marrow blast percentages at diagnosis $(P=0.003)$ than de novo individuals. They were less likely to be NPMI mutated $(P=0.03)$, to harbor a FLT3-ITD $(\mathrm{P}=0.02)$ and to be DNMT3A mutated $(P=0.04)$ but more likely to be JAK2 mutated ( $P=0.001$, Fig. 4 h). Within patients with adverse 
Table 2 Multivariate analyses for all patients.

\begin{tabular}{|c|c|c|c|c|c|c|}
\hline & \multicolumn{2}{|c|}{$\begin{array}{l}\text { Cumulative incidence of } \\
\text { relapse }\end{array}$} & \multicolumn{2}{|c|}{$\begin{array}{l}\text { Cumulative incidence of } \\
\text { non-relapse mortality }\end{array}$} & \multicolumn{2}{|l|}{ Overall survival } \\
\hline & $\operatorname{HR}^{\mathrm{a}}(95 \% \mathrm{CI})$ & $P$ & $\operatorname{HR}^{\mathrm{a}}(95 \% \mathrm{CI})$ & $P$ & $\mathrm{OR}^{\mathrm{b}}(95 \% \mathrm{CI})$ & $P$ \\
\hline $\begin{array}{l}\text { ELN2017 genetic risk } \\
\text { (adverse vs intermediate vs } \\
\text { favorable) }\end{array}$ & $\begin{array}{l}1.72 \\
(1.22-2.42)\end{array}$ & 0.002 & - & - & $\begin{array}{l}0.66 \\
(0.49-0.88)\end{array}$ & 0.006 \\
\hline Age at HSCT & - & - & $\begin{array}{l}1.03 \\
(1.02-1.05)\end{array}$ & $<0.001$ & - & - \\
\hline $\begin{array}{l}\text { Remission status at HSCT } \\
\text { (CR vs CRi) }\end{array}$ & - & - & $\begin{array}{l}0.46 \\
(0.29-0.73)\end{array}$ & 0.03 & - & - \\
\hline $\begin{array}{l}\text { Pre-HSCT MRD status } \\
\text { (positive vs negative) }\end{array}$ & $\begin{array}{l}3.22 \\
(1.89-5.48)\end{array}$ & $<0.001$ & - & - & $\begin{array}{l}0.54 \\
(0.34-0.87)\end{array}$ & 0.01 \\
\hline
\end{tabular}

Variables considered in the models were those significant at $\alpha=0.10$ in univariable analyses.

For cumulative incidence of relapse endpoint, variables considered were: ELN2017 genetic risk group, age at HSCT, and pre-HSCT MRD status conditioning regimen (RIC/MAC vs NMA).

For non-relapse mortality endpoint, variables considered were: disease origin (de novo vs s/tAML), age at HSCT, remission status at HSCT (CR vs CRi), conditioning regimen (RIC/MAC vs NMA), and donor type (mismatched vs matched unrelated vs related).

For OS endpoint, variables considered were: disease origin (de novo vs s/tAML), ELN2017 genetic risk group, age at HSCT, pre-HSCT MRD status, conditioning regimen (RIC/MAC vs NMA), remission status at HSCT (CR vs CRi) and donor type (mismatched vs matched unrelated vs related).

$A M L$ acute myeloid leukemia, $C I$ confidence interval, $C R$ complete remission, $C R i$ complete remission with incomplete peripheral recovery, ELN European LeukemiaNet, HSCT hematopoietic stem cell transplantation.

${ }^{a} \mathrm{HR}$, hazard ratio, $<1(>1)$ indicate lower (higher) risk of relapse for the first category listed for the dichotomous variables.

${ }^{\mathrm{b}} \mathrm{OR}$, odds ratio, $<1(>1)$ indicate lower (higher) chance of survival for the first category listed for the dichotomous variables.

risk according to ELN, s/tAML patients were older $(P=$ $0.008)$, had lower platelet counts $(P=0.03)$, lower bone marrow blast percentages $(P=0.006)$ and a higher CD34 $+/ \mathrm{CD} 38-$ cell burden at diagnosis $=(P=0.05)$ than de novo individuals. They were also less likely to harbor a FLT3-ITD $(P=0.03)$ and by trend FLT3-TKD $(P=0.09)$ and more likely to be ASXL1 mutated $(P=0.05$, Fig. 41$)$.

\section{Outcome of s/tAML patients within the three ELN risk groups}

The ELN risk groups have been shown to allow a separation of patients in risk groups with distinct outcomes [2, 3, 31] and were distributed significantly different between de novo and s/tAML patients. Subsequently, we analyzed the prognostic impact of s/tAML compared to de novo AML within the ELN risk groups separately. Within the group of favorable ELN risk ( $n=107$, Fig. $4 \mathrm{~b}, \mathrm{c})$, patients with s/ tAML $(n=17)$ had significantly higher CIR $(P=0.005)$, but comparable OS $(P=0.80)$ as de novo AML patients ( $n$ $=90$ ). Noteworthy is the low number of patients in the s/ tAML group with ELN favorable risk, suggesting the results to be interpreted with caution. In contrast, neither within the group with intermediate $(n=115$, Fig. $4 \mathrm{f}, \mathrm{g})$ nor adverse ELN risk ( $n=152$, Fig. 4 j, k) distinct outcomes according to disease origin were observed. Finally, also in the high- risk group of patients with detectable pre-HSCT MRD, no distinct CIR $(P=0.63)$ and OS $(P=0.40$, Supplementary Fig. S4) were observed.

\section{Discussion}

The here observed associations of s/tAML compared to de novo AML patients are in line with previously published data $[7-9,12,20]$. Our study also shows that ELN adverse risk is more frequent and ELN favorable risk less frequent in s/tAML (Fig. 1b) compared to de novo AML patients.

After consolidation chemotherapy, adverse outcomes for s/tAML compared to de novo individuals have been shown, but this difference is reduced in older individuals $(>60$ years) or when high-risk genetic subgroups were regarded separately [7-9]. After allogeneic HSCT, there are only limited and conflicting data comparing de novo and s/tAML $[19,20]$ and no study focused on older individuals, representing the majority of s/tAML patients, and within the context of the most recent ELN risk classification. Regarding all patients, we observed a shorter OS for s/ tAML patients which primarily was caused by higher NRM after allogeneic HSCT (Fig. 2b). Importantly, in multivariate analyses, after adjustment for ELN risk and preHSCT MRD status, disease origin did not impact CIR or 


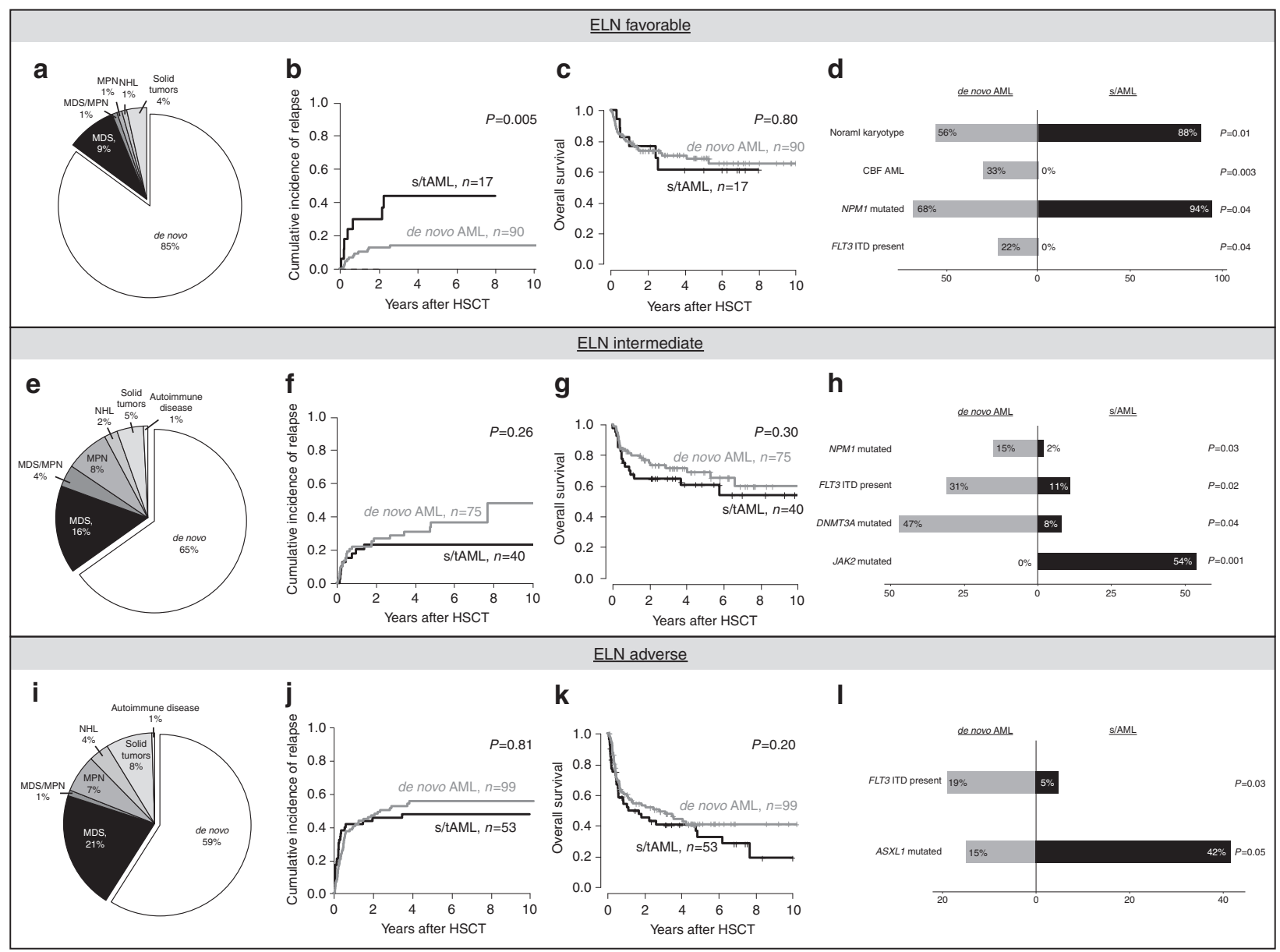

Fig. 4 Outcome and disease characteristics according to disease origin (de novo vs secondary or treatment-related AML) within the separate ELN risk groups. a Distribution of primary disease, b cumulative incidence of relapse, $\mathbf{c}$ overall survival, and $\mathbf{d}$ genetic associations in patients with favorable ELN risk, e distribution of primary disease, $\mathbf{f}$ cumulative incidence of relapse, $\mathbf{g}$ overall survival, and $\mathbf{h}$ genetic associations in patients with intermediate ELN risk and $\mathbf{i}$ distribution of primary disease, $\mathbf{j}$ cumulative incidence of relapse, $\mathbf{k}$ overall survival, and $\mathbf{l}$ genetic associations in patients with adverse ELN risk.
OS. In separate analyses according to the applied conditioning regimens, no outcome difference between de novo and s/tAML was seen after NMA conditioning. Only within patients receiving RIC- or MAC-HSCT, having s/tAML remained a prognostic factor for higher NRM in both univariate and multivariate analyses. However, only $28 \mathrm{~s} /$ tAML patients received RIC- or MAC-HSCT in the here analyzed set. Two other studies compared de novo and s/ tAML patients undergoing allogeneic HSCT [19, 20]. Overall outcomes in both studies match our analysis which is also true for the higher age and a more frequent use of lower intensity conditioning in the s/tAML cohorts. One other single centre analysis showed no distinct outcomes for patients transplanted in CR1/CRi1 [20]. In this study, lower patient numbers and no distinct cytogenetic risk between de novo and sAML patients may have contributed to the lacking outcome differences.

In contrast, the EBMT recently reported higher CIR and NRM and shorter OS for s/tAML patients, independently of conditioning intensity or cytogenetic risk [19]. Among the suggested reasons for these outcome differences was a lower ability to tolerate allogeneic HSCT-related toxicities in s/tAML patients. As we did not observe higher NRM or shorter OS after NMA-HSCT, but after RIC- or MACHSCT, our study contributes to this assumption of a predisposition to treatment-related complications after more intensive conditioning regimens in s/tAML patients. Another speculation of Schmaelter et al. was that a higher pre-HSCT MRD burden might have contributed to the worse outcomes [19]. We were able to assess the pre-HSCT MRD status (as previously described [26-28] based on NPM1 mutation status and BAALC and $M N 1$ expression) in 244 patients which did not differ between de novo or s/ tAML in our cohort, neither within the whole patient population (Supplementary Table S3) nor separately within the three ELN risk groups (Supplementary Table S5). As expected, MRD positivity correlated well with higher relapse probabilities which was seen irrespective of disease 
origin in both de novo and s/tAML patients (Supplementary Fig. S5) or conditioning regimen (Supplementary Fig. S6). In addition, outcome of pre-HSCT MRD positive patients was dismal and did not differ between de novo or s/tAML groups (Supplementary Fig. S4).

After consolidation chemotherapy, outcome differences between s/tAML and de novo AML patients have also been shown within different genetic risk groups $[9,32]$ but were reported to be larger in patients with favorable rather than adverse or intermediate genetic risk [12]. To our knowledge, we are the first to report on s/tAML patients receiving HSCT in the context of the most recent ELN risk classification, which relies to a larger extend on the molecular disease characterization [1]. Within the three ELN risk groups, between de novo and s/tAML patients, we observed a significantly different distribution of genetic characteristics, indicating distinct genetic drivers of the disease (Fig. 4d, h, i). In the ELN favorable group, s/tAML patients had less FLT3-ITD and CBF AML than de novo AML patients. Subsequently, and in contrast to the whole patient cohort, in ELN favorable risk the amount of patients with a normal karyotype or a NPMI mutation was higher in s/ tAML than de novo AML patients. Within the ELN intermediate group, s/tAML patients were more likely to be $J A K 2$ mutated but less likely to be NPM1, DNMT3A, or FLT3-ITD mutated. In ELN adverse risk, s/tAML patients again had less FLT3-ITD, but were more often ASXL1 mutated, which has been linked to sAML [33]. Regarding outcomes, only within the ELN favorable risk group we observed a higher CIR for s/tAML patients, likely driven by the lower incidence of CBF AML. CIR and OS remained similar between de novo and s/tAML patients within the ELN intermediate and adverse groups. Our data suggest that when the ELN risk groups are considered, no distinct survival can be shown between de novo or s/tAML patients receiving HSCT in remission and, thus, that allogeneic HSCT might contribute to better outcomes in this patient population.

Recently, a variety of new substances have been introduced into AML treatment. CPX-351 has been shown to improve outcomes for patients with s/tAML as compared to standard $7+3$ chemotherapy [34]. Combination therapies of standard $7+3$ with FLT3 inhibitors, as Midostaurin, in patients with FLT3-mutated AML [35] or gemtuzumab ozogamicin (GO) in CD33-positive favorable or intermediate risk AML [36] represent a new standard of care. Of note, none of the patients in our set received CPX-351 or GO and all patients treated within FLT3 inhibitor studies had de novo disease as prior chemotherapies were excluded according to study protocols. We also found lower CD33 expression levels in s/tAML compared to de novo AML patients (Supplementary Fig. S7), which might indicate reduced efficacy of GO in these patients and raises the question of applying higher dosages in selected patients. How these new substances will fit into the treatment of s/ tAML patients remains to be elucidated, but treatment combinations of CPX-351 with GO (ClinicalTrials.gov Identifier: NCT03904251) and/or FLT3 inhibitors (NCT04128748) in eligible patients will likely further improve outcomes in s/tAML patients.

Relevant limitations of our study are the retrospective nature and restricted patient numbers within some subgroup analyses, including e.g., patients receiving RIC- or MACHSCT. In addition, only a restricted number of patients had the pre-HSCT MRD status available as well as could be comprehensively molecularly characterized for their ASXL1, RUNX1, and TP53 mutation status-relevant for the genetic risk classification according to ELN2017 at diagnosis, leading to restricted patient numbers in some subgroups. Especially the s/tAML ELN2017 favorable subgroup included only 17 patients in the outcome analysis.

In conclusion, consistent with previous studies $[19,20]$, our data show that allogeneic HSCT is a feasible and often curative consolidation option for s/tAML patients. While s/tAML patients were older and more likely to harbor adverse ELN risk, outcomes between de novo and s/tAML patients did not differ when these covariables were considered. Thus, not all s/tAML patients have a dismal prognosis when undergoing allogeneic HSCT. Pre-HSCT MRD positivity remained an important prognostic factor in both de novo and s/tAML patients and showed no distinct incidence between both patient populations. These data highlight the importance of the ELN2017 classification and pre-HSCT MRD status for risk stratification also in s/tAML.

Acknowledgements This study was supported by the Verein Zusammen gegen den Krebs e.V, (SS), the Deutsche Jose-Carreras-Stiftung (04R/2016 [SSch] and PS15/05 [JG]), the Deutsche Gesellschaft für Innere Medizin (Clinician Scientist Program [MJ]) and a Novartis Research Grant (HRYD-030 [SS]). The authors thank Christel Müller, Daniela Bretschneider, Evelin Hennig, Sabine Leiblein, Martina Pleß, Ulrike Bergmann, Janet Bogardt, Annette Jilo, and Dagmar Cron for their help in determine cytogenetic, morphologic and immunological analyses, and Christine Günther, Scarlett Schwabe, Ines Kovacs, and Kathrin Wildenberger for their help in sample processing.

Author contributions $\mathrm{MJ}$ and SS contributed to the design and analysis of this study and the writing of the manuscript, and all authors agreed on the final version. MJ, JG, MB, DBr, Dba, KG, and JS carried out the laboratory-based research. MJ and SS performed statistical analyses. UP, DN, and SS provided administrative support.

\section{Compliance with ethical standards}

Conflict of interest The authors declare that they have no conflict of interest. 
Publisher's note Springer Nature remains neutral with regard to jurisdictional claims in published maps and institutional affiliations.

Open Access This article is licensed under a Creative Commons Attribution 4.0 International License, which permits use, sharing, adaptation, distribution and reproduction in any medium or format, as long as you give appropriate credit to the original author(s) and the source, provide a link to the Creative Commons license, and indicate if changes were made. The images or other third party material in this article are included in the article's Creative Commons license, unless indicated otherwise in a credit line to the material. If material is not included in the article's Creative Commons license and your intended use is not permitted by statutory regulation or exceeds the permitted use, you will need to obtain permission directly from the copyright holder. To view a copy of this license, visit http://creativecommons. org/licenses/by/4.0/.

\section{References}

1. Döhner H, Estey E, Grimwade D, Amadori S, Appelbaum FR, Ebert BL, et al. Diagnosis and management of AML in adults: 2017 ELN recommendations from an international expert panel. Blood. 2017;129:424-47.

2. Herold T, Rothenberg-Thurley M, Grunwald VV, Janke H, Goerlich D, Sauerland MC et al. Validation and refinement of the revised 2017 European LeukemiaNet genetic risk stratification of acute myeloid leukemia. Leukemia. 2020. https://doi.org/10.1038/ s41375-020-0806-0.

3. Grimm J, Jentzsch M, Bill M, Goldmann K, Schulz J, Niederwieser D et al. Prognostic Impact of the European LeukemiaNet 2017 Risk classification in acute myeloid leukemia patients receiving allogeneic transplantation. Blood Adv. 2020;4: 3864-74.

4. Schuurhuis GJ, Heuser M, Freeman S, Béne MC, Buccisano F, Cloos $\mathrm{J}$, et al. Minimal/measurable residual disease in AML: a consensus document from the European LeukemiaNet MRD Working Party. Blood. 2018;131:1275-91.

5. Larson RA. Cytogenetics, not just previous therapy, determines the course of therapy-related myeloid neoplasms. J Clin Oncol. 2012;30:2300-2.

6. Morton LM, Dores GM, Tucker MA, Kim CJ, Onel K, Gilbert ES, et al. Evolving risk of therapy-related acute myeloid leukemia following cancer chemotherapy among adults in the United States, 1975-2008. Blood. 2013;121:2996-3004.

7. Kayser S, Do K, Ko C, Horst HA, Held G, Lilienfeld-toal MVon, et al. The impact of therapy-related acute myeloid leukemia (AML) on outcome in 2853 adult patients with newly diagnosed AML. Blood. 2011;117:2137-46.

8. Granfeldt Østgård LS, Medeiros BC, Sengeløv H, Nørgaard M, Andersen MK, Dufva I, et al. Epidemiology and clinical significance of secondary and therapy-related acute myeloid leukemia: a national population-based cohort study. J Clin Oncol. 2015;33:3641-9.

9. Hulegårdh E, Nilsson C, Lazarevic V, Garelius H, Antunovic P, Rangert Derolf $\AA$, et al. Characterization and prognostic features of secondary acute myeloid leukemia in a population-based setting: a report from the Swedish Acute Leukemia Registry. Am J Hematol. 2015;90:208-14.

10. Mesa RA, Li CY, Ketterling RP, Schroeder GS, Knudson RA, Tefferi A. Leukemic transformation in myelofibrosis with myeloid metaplasia: A single-institution experience with 91 cases. Blood. 2005;105:973-7.

11. Rogers HJ, Wang X, Xie Y, Davis AR, Thakral B, Wang SA, et al. Comparison of therapy-related and de novo core binding factor acute myeloid leukemia: a bone marrow pathology group study. Am J Hematol. 2020;95:799-808.

12. Schoch C, Kern W, Schnittger S, Hiddemann W, Haferlach T. Karyotype is an independent prognostic parameter in therapyrelated acute myeloid leukemia (t-AML): An analysis of 93 patients with t-AML in comparison to 1091 patients with de novo AML. Leukemia. 2004;18:120-5.

13. Kröger N, Eikema D, Köster L, Beelen D, de Wreede LC, Finke J, et al. Impact of primary disease on outcome after allogeneic stem cell transplantation for transformed secondary acute leukaemia. $\mathrm{Br}$ J Haematol. 2019;185:725-32.

14. Kröger N, Brand R, Van Biezen A, Zander A, Dierlamm J, Niederwieser $\mathrm{D}$, et al. Risk factors for therapy-related myelodysplastic syndrome and acute myeloid leukemia treated with allogeneic stem cell transplantation. Haematologica. 2009;94:542-9.

15. Lee CJ, Labopin M, Beelen D, Finke J, Blaise D, Ganser A, et al. Comparative outcomes of myeloablative and reduced-intensity conditioning allogeneic hematopoietic cell transplantation for therapy-related acute myeloid leukemia with prior solid tumor: a report from the acute leukemia working party of the European societ. Am J Hematol. 2019;94:431-8.

16. Finke J, Schmoor C, Bertz H, Marks R, Wasch R, Zeiser R, et al. Long-term follow-up of therapy-related myelodysplasia and AML patients treated with allogeneic hematopoietic cell transplantation. Bone Marrow Transpl. 2016;51:771-7.

17. Litzow MR, Tarima S, Pérez WS, Bolwell BJ, Cairo MS, Camitta $\mathrm{BM}$, et al. Allogeneic transplantation for therapy-related myelodysplastic syndrome and acute myeloid leukemia. Blood. 2010;115:1850-7.

18. Sengsayadeth S, Gatwood KS, Boumendil A, Labopin M, Finke J, Ganser A, et al. Conditioning intensity in secondary AML with prior myelodysplastic syndrome/myeloproliferative disorders: An EBMT ALWP study. Blood Adv. 2018;2:2127-35.

19. Schmaelter AK, Labopin M, Socié G, Itälä-Remes M, Blaise D, Yakoub-Agha I, et al. Inferior outcome of allogeneic stem cell transplantation for secondary acute myeloid leukemia in first complete remission as compared to de novo acute myeloid leukemia. Blood Cancer J. 2020;10:10-26.

20. Michelis FV, Atenafu EG, Gupta V, Kim DD, Kuruvilla J, Lipton $\mathrm{JH}$, et al. Comparable outcomes post allogeneic hematopoietic cell transplant for patients with de novo or secondary acute myeloid leukemia in first remission. Bone Marrow Transpl. 2015;50:907-13.

21. Zeijlemaker W, Grob T, Meijer R, Hanekamp D, Kelder A, Carbaat-Ham JC, et al. CD34+CD38- leukemic stem cell frequency to predict outcome in acute myeloid leukemia. Leukemia. 2019;33:1102-12.

22. Arber DA, Orazi A, Hasserjian R, Thiele J, Borowitz MJ, Le Beau $\mathrm{MM}$ et al. The 2016 revision to the World Health Organization classification of myeloid neoplasms and acute leukemia. Blood. 2016;127:2391-405.

23. Bill M, Jentzsch M, Grimm J, Schubert K, Lange T, Cross M, et al. Prognostic impact of the European LeukemiaNet standardized reporting system in older AML patients receiving stem cell transplantation after non-myeloablative conditioning. Bone Marrow Transpl. 2017;52:932-5.

24. Jentzsch M, Bill M, Grimm J, Schulz J, Schuhmann L, Brauer D et al. High expression of the stem cell marker GPR56 at diagnosis identifies acute myeloid leukemia patients at higher relapse risk after allogeneic stem cell transplantation in context with the CD34+/CD38 - population. Haematologica. 2020;105:229260

25. Grimm J, Bill M, Jentzsch M, Beinicke S, Häntschel J, Goldmann $\mathrm{K}$ et al. Clinical impact of clonal hematopoiesis in acute myeloid 
leukemia patients receiving allogeneic transplantation. Bone Marrow Transplant. 2019;54. https://doi.org/10.1038/s41409-0180413-0.

26. Jentzsch M, Bill M, Grimm J, Schulz J, Beinicke S, Häntschel J, et al. Prognostic impact of blood MN1 copy numbers before allogeneic stem cell transplantation in patients with acute myeloid leukemia. HemaSphere. 2019;3:e167.

27. Bill M, Grimm J, Jentzsch M, Kloss L, Goldmann K, Schulz J et al. Digital droplet PCR-based absolute quantification of pretransplant NPM1 mutation burden predicts relapse in acute myeloid leukemia patients. Ann Hematol. 2018;97. https://doi.org/10. 1007/s00277-018-3373-y.

28. Jentzsch M, Bill M, Grimm J, Schulz J, Goldmann K, Beinicke S et al. High BAALC copy numbers in peripheral blood prior to allogeneic transplantation predict early relapse in acute myeloid leukemia patients. Oncotarget 2017;8. https://doi.org/10.18632/ oncotarget. 21322.

29. R Development Core Team. R: a language and environment for statistical computing. R Foundation for Statistical Computing. Vienna, Austria: R Development Core Team; 2017. ISBN 3900051-07-0. http://www.R-project.org.

30. Breems DA, Van Putten WLJ, De Greef GE, Van Zelderen-Bhola SL, Gerssen-Schoorl KBJ, Mellink CHM, et al. Monosomal karyotype in acute myeloid leukemia: a better indicator of poor prognosis than a complex karyotype. J Clin Oncol. 2008;26:4791-7.
31. Harada Y, Nagata Y, Kihara R, Ishikawa Y, Asou N, Ohtake S, et al. Prognostic analysis according to the 2017 ELN risk stratification by genetics in adult acute myeloid leukemia patients treated in the Japan Adult Leukemia Study Group (JALSG) AML201 study. Leuk Res. 2018;66:20-27.

32. Koh Y, Kim I, Bae JY, Song EY, Kim HK, Yoon SS, et al. Prognosis of secondary acute myeloid leukemia is affected by the type of the preceding hematologic disorders and the presence of trisomy 8. Jpn J Clin Oncol. 2010;40:1037-45.

33. Lindsley RC, Mar BG, Mazzola E, Grauman PV, Shareef S, Allen SL, et al. Acute myeloid leukemia ontogeny is defined by distinct somatic mutations. Blood. 2015;125:1367-76.

34. Lancet JE, Uy GL, Cortes JE, Newell LF, Lin TL, Ritchie EK, et al. Cpx-351 (cytarabine and daunorubicin) liposome for injection versus conventional cytarabine plus daunorubicin in older patients with newly diagnosed secondary acute myeloid leukemia. J Clin Oncol. 2018;36:2684-92.

35. Stone RM, Mandrekar SJ, Sanford BL, Laumann K, Geyer S, Bloomfield CD, et al. Midostaurin plus chemotherapy for acute myeloid leukemia with a FLT3 Mutation. N. Engl J Med. 2017;377:454-64.

36. Hills RK, Castaigne S, Appelbaum FR, Delaunay J, Petersdorf S, Othus $\mathrm{M}$, et al. The addition of gemtuzumab ozogamicin to induction chemotherapy in acute myeloid leukaemia: an individualpatient data meta-analysis of randomised trials in adults. Lancet Oncol. 2014;15:986-96. 